\title{
A Study On The Construction Of Virus Infection Area Information System Using Iomt Environment
}

\author{
Sungho Sim ${ }^{1}$, Hanyong Choi*2 \\ ${ }^{1}$ College of General Education, Semyung University.,Jecheon, Republic of Korea \\ ${ }^{*}$ Division of IT Convergence Engineering, Shinhan University.,Dongducheon, Republic of Korea \\ shshim@ semyung.ac.kr ${ }^{1}$, hychoi@ shinhan.ac.kr ${ }^{2}$
}

Article History: Received: 11 January 2021; Accepted: 27 February 2021; Published online: 5 April 2021

\begin{abstract}
With the recent emergence of novel virus, the importance has been empathized on the epidemiologic investigation of the infection route and control of infected patients. Control system on the viral infections is required since the new virus expression cycle becomes faster which was not previously identified with the spread to other regions and countries. Viral infections cause multiple types of problems such as public health, economic and social ones. In addition, viruses can be spread fast beyond the regions and countries all over the world according to the movements of population. Although national quarantine systems have been established to solve these problems by countries, earlier actions on the novel virus were not satisfactory. IoT environment-based healthcare industry and IT convergence medical service industry have been continuously growing. Healthcare IT, which is the area to deal with information on the personal health and medical cares, devices, systems, and platforms, provides with personal healthcare services. In this article, information system on the virus infected territories is proposed using Internet of Medical Things (IoMT) environment. The proposed study is to establish the information system on the virus infected territories by IoMT-based monitoring of infection symptoms for early reactions against the viral infections. The system can be reacted proactively by detecting the risk factors of community spread from early detections of infected patients under the fast spread situation of viral diseases. The information system on the virus infected territories using IoMT environment can react to the spread of infectious diseases actively upon providing the information of infectious disease symptoms using user information. The proposed system can establish the integrative control system of viral diseases by the prediction module of community outbreak and spread of viral diseases.
\end{abstract}

Keywords: ICT, IoMT, IoT, Virus, Disease.

\section{Introduction}

Despite the development of modern medicine, communicable diseases have not been disappeared but emerged continuously. Communicable diseases which had been disappeared in the past were appeared again and emergences of new communicable diseases threaten the health of mankind [1]. Recently, viral communicable diseases with fast spread and higher pathogenicity have been increased over the world. Communicable diseases mean the diseases infecting from human to human, causing from specific pathogens [2]. Pathogens of communicable diseases include bacteria, Spirochetes, Rickettsia, viruses, fungi, and so on [3]. World Health Organization classifies H1N1 and highly pathogenic Avian Influenza as the communicable diseases with high infectivity and frequent modifications [4]. For legal communicable diseases, their spread to others are regulated by policies and regulations on preventions at the national level. To prevent the spread of communicable diseases, most countries predict and prepare the spread of the diseases using mathematical models [5-7]. With the 4th industrial revolution, multiple studies have been conducted on convergence technologies with medical and IT. Medical-IT convergence, developing new value from convergence of medical field into IT, has been progressed into the medical services to manage the health and prevent diseases without any limitations on time and space. It has been changed into the new forms of enhancing efficiency and enabling treatments, managements, and preventions of diseases upon integrating IT such as healthcare using IoT, medical dBASE, digital hospitals, remote medical services, and so on, with medical devices [8-10]. Medical-IT convergence enables intelligent medical services using medical information, efficiently. Further studies on the intelligent and remote medical services are required to manage the new projects of medical services and increasing communicable diseases. Communicable disease information system is required using monitoring for these service supports. Using ICT, Smart home, and IoT devices, required information can be collected by monitoring users' daily living environment [11,12]. Developments of wearables, sensors, big data, IoT devices, network, and so on lead the innovation of medical field to establish new foundation. By capture, generation, analysis, and transmission of data, IoMT environment is generated upon connecting with medical devices, medical services, and medical software [13,14].

Prevention and management methods proper for the users can be provided by collecting users' biological information using IoMT. Also, real-time monitoring users can make proper medical supporting services [15,23]. This article suggests information system on the virus infected territories using IoMT environment. The proposed system collects the user information using devices under the IoMT environment. Collected information provides users with stepwise information messages upon comparisons with symptoms of existing viral diseases from 
matching with information of legal communicable diseases. New virus information can be updated by monitoring, and viral infection can be prevented for users through user location-based informing of infection occurrences and risky areas in case of the occurrences of infected patients. The proposed system can reduce the spread of communicable diseases into the community by providing user-centric virus information beyond the existing passive delivery of information.

\section{Related Works}

\subsection{IoMT}

IoMT, which is the area to deal with information on the personal health and medical cares, devices, systems, and platforms, can provide users with customized healthcare services under the online environment. IoMT can enhance the efficiency by integrating medical devices with intelligent data analysis under IoT environment and improve service qualities and service accessibility by remote monitoring, real-time user management, and customized service provisions. It can provide medical supports whenever and wherever based on on-demand healthcare services. Public health IoT can support the treatments and preventive measures to meet the situation by monitoring personal health and medical information. That means IoMT can be defined as the integration of medical devices and application programs to support connection into the medical information technology system using networking technology [16,24]. Figure 1 shows the model of IoMT.

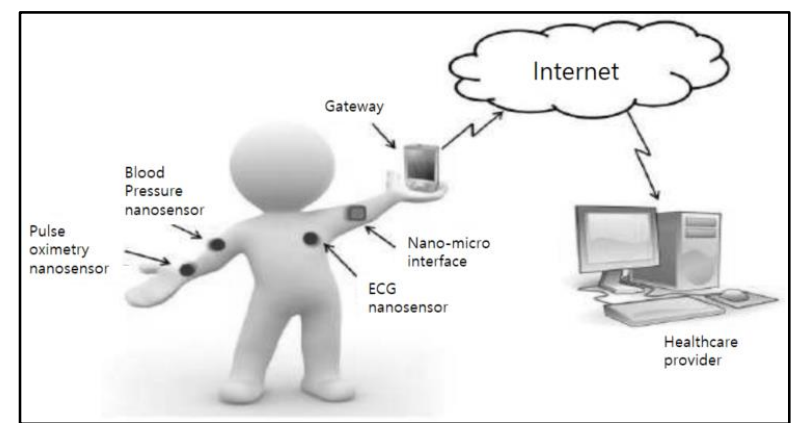

Figure 1. Internet of medical things model

IoMT uses numerous sensors and actuators to support the services according to the users' situations. It provides the optimized medical support services by using smart devices and collecting usable information through integrative networking function. These devices can access the open network services, and data can be collected by monitoring from information providers online worldwide. User information can be collected by wearables, finess shoes, RFIDbased devices, sensors, and so on [17]

\subsection{IoMT Devices}

IoMT devices can collect biological signals. General biological signals including Photoplethysmogram (PPG), Electromyogram (EMG), Electroencephalogram (EEG), movement data, and body sounds can be collected by monitoring IoMT devices. Table 1 shows four signals generally used [18].

Table 1. Frequency and common biomedical signals

\begin{tabular}{|c|c|}
\hline Signal & $\begin{array}{c}\text { Frequency } \\
\text { Range }(\mathrm{Hz})\end{array}$ \\
\hline $\begin{array}{c}\text { Photoplethysmogr } \\
\text { am }\end{array}$ & $0.5-5^{1}$ \\
\hline Electromyogram & $50-150^{1}$ \\
\hline $\begin{array}{c}\text { Cardiac } \\
\text { Auscultation }\end{array}$ & $20-420^{1}$ \\
\hline Gait Analysis & $0-15^{1}$ \\
\hline
\end{tabular}

Recently, a variety of commercial wearable sensors can be used to monitor multiple biological signals remotely. Figure 2 shows IoMT devices [19]. Movement detecting sensor can detect the physical activities, collect real-time information of the sensor, and transmit it by operation with accelerometer [20]. Pressure sensor provides the range of blood pressure and expanded blood pressure, and monitors blood pressure and pulse rate. Temperature sensor monitors the change of body temperature at a real-time basis to be able to analyze infection, fever, inflammation, and hypothermia [21]. GPS Smartsoles can detect abnormal situations such as Alzheimer's diseases, dementia, other cognitive disorders, and so on [22]. Smart clothing monitors the users' status by measuring biological signals such as emotion and attaching sensor to follow up pulse rate. 




Figure 2. loMT Devices

\section{Proposed method}

Lots of people are threatened and significant amount of social burdens for quarantine are required due to viral diseases. Also, they affect the economic activities in the communities and country. System establishment is required to prevent $n$-times infection and lower spread of infectious diseases by early quarantine and epidemiologic investigation in case of viral disease outbreak. In this section, information system on the virus infected territories is proposed using IoMT environment.

\subsection{Virus Infected Area Information System Model}

Information system on the virus infected territories using IoMT environment provides potential users for infections with information services including visit records of infected patients in the related regions in case of the occurrences of infected patients. Probability of viral infection can be reduced by guiding avoidance of infection risk areas. Also, the information system collects the information of legal communicable diseases as well as the monitoring information on the new viral diseases occurred in the country and regions. The system can reduce the spread of viral infectious diseases providing stepwise information messages to meet the symptoms of viral infections from matching user information and providing infection information and safe movement routes. The information system on the virus infected territories is a model to establish the information on the communicable diseases, to investigate the transmission routes of the diseases and to lower the spread. Additional spread can be prevented by understanding of transmission routes and infection potential can be excluded by avoidance of infection risk areas by sharing the information on the infected territories. The information system collects the user information using IoMT devices. Information services by grades can be provided upon matching the collected user information with the information of legal communicable diseases. Information on the symptoms of infectious diseases is provided by matching with users' symptoms in case of emergences of new infectious diseases upon confirming the information on the new diseases generated by monitoring agent of disease information and comparing with users' symptoms. Figure 3 shows IoT environment-based information system on the virus infected territories.

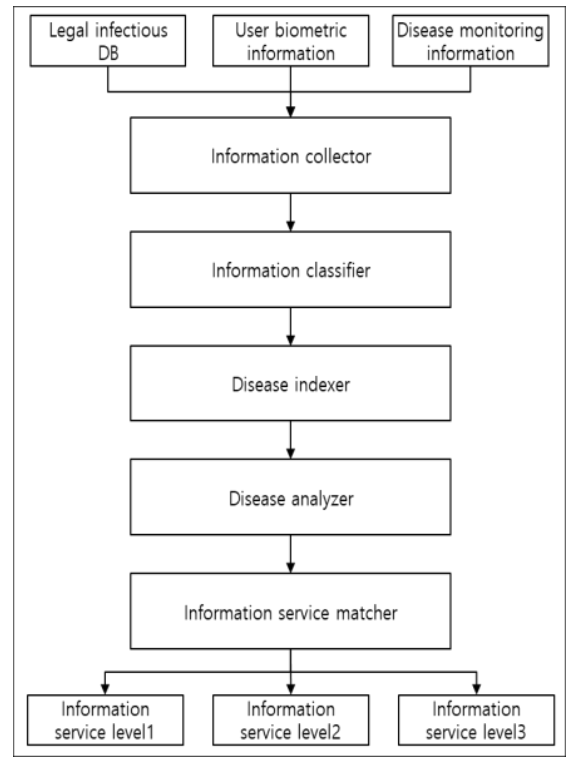

Figure 3. Virus infection area information system structure 


\subsection{Collection information model}

The proposed system collects the information of legal communicable diseases, users, and disease outbreak monitoring to establish the information on the virus infected territories. Information of legal communicable diseases is collected to search the symptoms of existing diseases upon comparisons with users' symptoms. Users' biological information is collected using IoMT devices. Values on the changes of users' biological information are measured upon collecting the information on fever, respiratory symptoms, users' behaviors, and environment. For disease monitoring information, data are collected from local and national information providers using agents. In case of new viral disease outbreak, early reactions are possible and spread of viral diseases can be delayed using monitoring information. Figure 4 shows the model of information system on the virus infected territories.

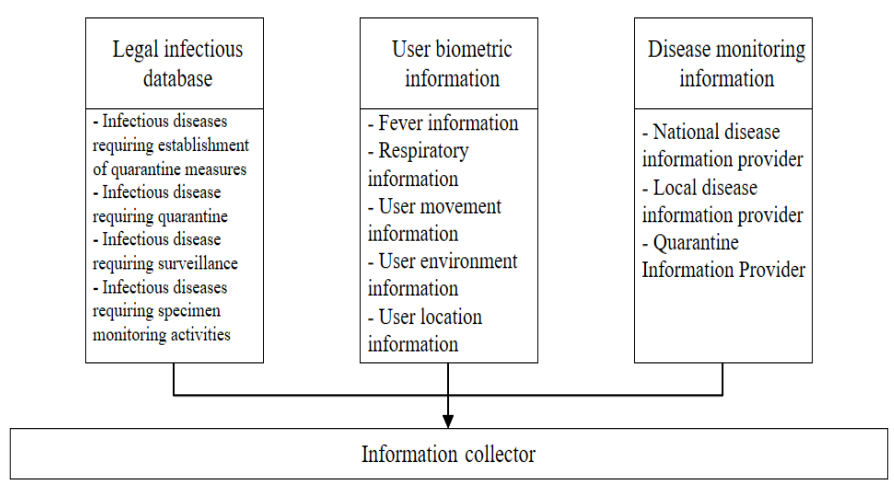

Figure 4. Collection information model

Collected information from information collector is the infectious disease required for the establishment of quarantine measures, for isolation, for monitoring, and for sample monitoring activities from the database of legal communicable diseases. Collected users' biological information includes fever, respiratory symptoms, behaviors, environment, and location of the users. Also, disease information is monitored by national disease information providers, local disease information providers, and quarantine information providers.

\subsection{Virus-infected area information system matching procedure}

In the proposed system, information system on the infected territories is established by adding users' biological information and collected information from disease monitoring agents based on the existing disease information system. Figure 5 shows the matching process using legal disease information.

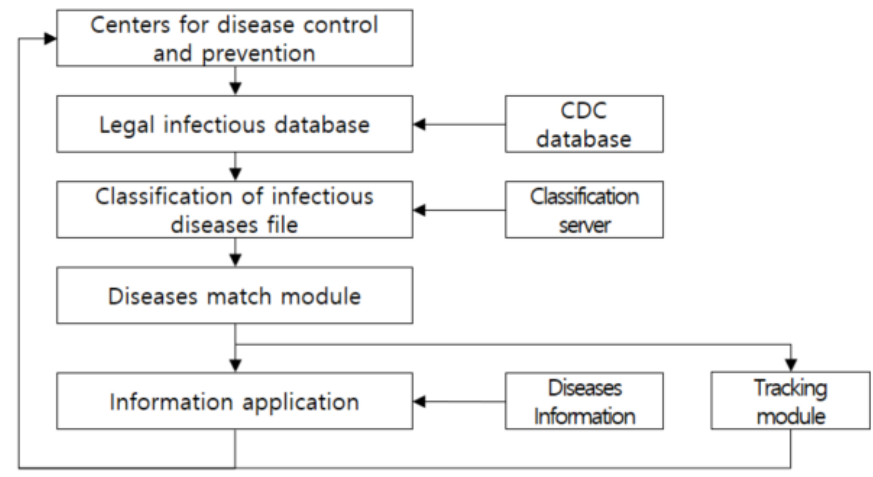

Figure 5. Matching procedure using legal illness information

A user accesses the center for disease control (CDC). The user checks the registered information from database of legal infectious diseases in CDC. Information on the infectious diseases by grades is acquired by classified disease files in the classification sever. Disease matching module performs the matching process with searching keywords of users and CDC registered database and provides the results to information application and tracking module. Information application shows the user matching results. Tracking module tracks the information on the repetitive symptoms and infectious diseases, providing CDC with matched information. Figure 6 shows the matching process using users' biological information and disease monitoring information. 


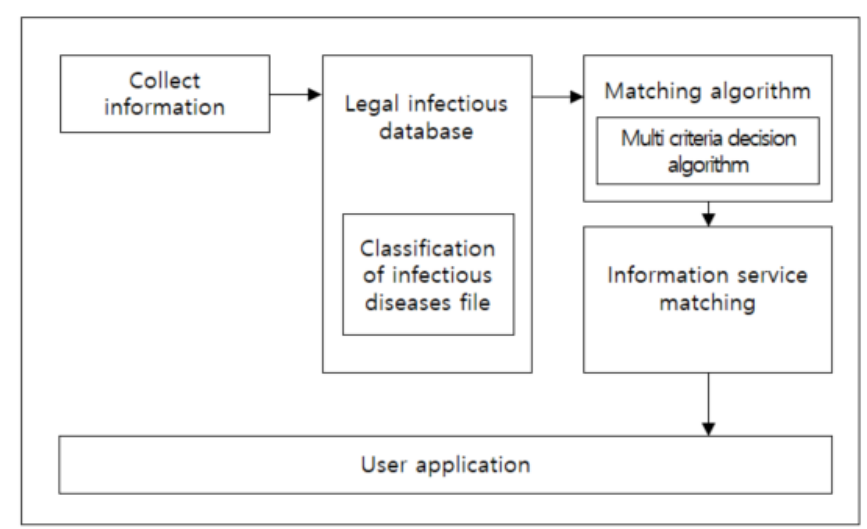

Figure 6. Matching procedure using user's biometric information and disease monitoring information

\section{Comparison analysis}

Social and economic problems have been increased due to faster emergences of new viruses and their outbreaks to other regions and countries. To solve these problems, control system on the viral infectious diseases and studies to prevent from their spread are required. CDC provides the services including information delivery on the viral infectious diseases timely after the outbreaks of pandemic viral diseases. This information delivery is effective for the existing registered diseases and viruses, however, it is difficult to cope with the emergences of new infectious diseases and novel viruses, effectively.

It may be difficult to countermeasure against the spread earlier if the symptoms are similar to those in the existing diseases or depending on the information providers.

In this article, information system on the virus infected territories using IoMT is proposed by addition of the information of users' symptoms and monitoring on the diseases in the country and territories. It was aimed to react against the spread of infectious diseases earlier not by passive way of information acquisition but by active way. The proposed system matches the symptoms of the diseases with those from legal communicable diseases expanding from passive type of information delivery into users' biological information and local disease monitoring information. Based on the matching information, stepwise information supporting service is provided to users. Early countermeasures against the spread within the community are possible by information delivery services in case of occurrences of infectious diseases. Also, movement routes of viruses can be tracked by accurate epidemiologic investigation through the location information of users. Table 2 shows the comparisons of the existing disease information system with the proposed one.

Table 2. Comparative analysis of proposed system

\begin{tabular}{|c|c|c|}
\hline $\begin{array}{c}\text { Informatio } \\
\text { n acquisition }\end{array}$ & Existing system & Propose method \\
\hline $\begin{array}{c}\text { Manageme } \\
\text { nt }\end{array}$ & Partial support & $\begin{array}{c}\text { IoMTdevice, } \\
\text { monitoring }\end{array}$ \\
\hline $\begin{array}{c}\text { Tracking } \\
\text { information }\end{array}$ & Not support & Partial support \\
\hline Monitoring & Not support & support \\
\hline
\end{tabular}

Information acquisition method of the existing system uses the registered information of users based on the regulations. The proposed system acquires the information from IoMT devices and disease monitoring agents. With respect to the post-hoc management, existing system is partly possible while the proposed system supports it by continuous disease information services. For follow up information, existing system relies on the epidemiologic investigation only causing ambiguous cases, however, the proposed system can track the viral movement routes partly using users' location and biologic information based on IoMT devices 


\section{Conclusion}

Recently, viral infectious diseases have been increased with high communicability. Unlike the past cases, the speed to spread among countries and regions is very fast. Until the treatments or vaccines are available in case of new virus outbreaks, multiple types of problems are imposed including economic, social ones, and so on. Quarantine systems on the infectious diseases by countries are established, however, most of them are focused on the countermeasures after outbreaks of communicable diseases. Active response system is required in case of outbreaks of viral infectious diseases. In this article, establishment of information system on the virus infected territories is proposed using IoMT environment. The proposed system collects user information using IoMTbased devices and wearables. Collected information provides with stepwise service messages upon comparisons with existing viral symptoms by matching information of legal communicable diseases designated by the country. New virus information is applied using monitoring agents and information on the infected territories is established using users' location-based information.

Probability to spread viral infections can be lowered by providing the information of infection occurrence and the risky areas of infections through information on the infected territories. Also, infection routes can be identified and avoiding routes of infected territories can be provided by epidemiologic investigation. The proposed system can prevent from spread among countries and regions by early countermeasures in case of occurrences of viral infectious diseases.

\section{References}

1. Choi, J.J. (2008). Development and Evalution of a Legal communicable Disease Electronic System for Infection control. Journal of Korean Academy of Fundamental Nursing, 15(3), 371-379.

2. Kim, E.G., Lee, S., Young, Y.B., Lee, H.J. \& Lee, T.J. (2013). Implementation of integrated monitoring system for trace and path prediction of infectious disease. Journal of Korean society for internet information, 14(5), 69-76.

3. Susan, S. \& Timothy R. Shope. (2013). Managing infectious diseases in child care and schools. 3rd Edition, American Academy of pediatrics, pp.169-171.

4. World Health Organization (WHO), Influenza virus activity in the world on September 2, 2012.

5. Towers, S., Geisse K.V., Tasl, C.C., Han, Q. \& Feng, Z. (2012). The impact of school closures on pandemic influenza: Assessing potential repercussions using a seasonal SIR model. Math. Biosci. Eng., 9(2), 413-430.

6. Lee, S., Golinski M. \& Chowell, G. (2012). Modeling optimal age-specific vaccination strategies against pandemic influenza. Bull. Math. Biol., 74, 958-980.

7. Paleshi, A., Evans, G.W., Heragu, S.S. \& Moghaddam, K.S. (2011). Simulation of mitigation strategies for a pandemic influenza. In Proceedings of the 2011 Winter simulation conference(WSC), 1340-1348..

8. Kim, H.S. (2017). A study on Usability improvement of mobile healthcare services. International Journal of advanced Smart Convergence(IJASC), 6(2), 72-81.

9. Lee, J.Y. \& Jung, K.D. (2016). Proposed Architecture for U-Healthcare System. International Journal of Advanced Culture Technology(IJACT), 4(2), 43-46.

10. Cho, B.H. (2018). Design of U-healthcare system for real-time blood pressure monitoring.The Journal of the institute of internet, broadcasting and communication(IIBC), 18(4), 161-168.

11. Muñoz, A., Augusto, J.C., Villa, A. \& Botía, J.A. (2011). Design and evaluation of an ambient assisted living system based on an argumentative multi-agent system, Personal and Ubiquitous Computing, 15(4), 377-387.

12. Tolentino, R.S., Lee, K.J., Kim, Y.T., Park, B.J. \& Park, G.C. (2010). A design of home-based monitoring U-healthcare system over ubiquitous sensor network. International Journal of Internet, Broadcasting and Communication(IJIBC), 2(1), 11-15.

13. Medtech and the internet of medical things, 2018. https://www2.deloitte.com/global/en/pages/lifesciences-and-healthcare/articles/medtech-internet-of-medical-things.html.

14. Hur, J.Y., Lee, K.Y., Lee, D.H. \& Kang, J.J. (2015) Design and development of smart monitoring system for u-healthcare. The Journal of the Convergence on Culture Technology(JCCT), 1(4), 107111.

15. N. Patel, "Internet of things in healthcare: applications, Benefits, and challenges," 2019. https://www.peerbits.com/blog/internet-of-things-healthcare-applications-benefits-andchallenges.html.

16. Frost\&Sullivan, "Internet of Medical Things(IoMT) Revolutionizing Healthcare, April 2017.

17. Polu, S.K. (2019). IoMT based smart health care monitoring system. International Journal for Innovative Research in Science \& Technology, 5(11), 58-64. 
18. Qureshi, F. \& Krishnan, S. (2018). Wearable hardware design for the internet of medical things(IoMT). Sensors, 18(11), 1-21.

19. Syed, L., Jabeen, S., Manimala, S. \& Alsaeedi, A. (2019). Smart healthcare framework for ambient assisted living using IoMT and big data analytics thechniques. Future Generation Computer System, 101. 136-151.

20. Eduardo Freitas, A.A. (2016). Wireless biomedical sensor networks: The technology. Proceedings of the 2nd World Congress on Electrical Engineering and Computer Systems and Science, 2016.

21. An, B.W., Shin, J.H., Kim, S.Y., Kim, J., Ji, S., Park, J. et al. Smart sensor systems for wearable electronic devices. Polymers, 9(12).

22. Vorp, G. (2015). Gps smart satellite monitoring and real -time tracking sole. 2015.

23. Maake, B. M., \&Tranos, Z. U. V. A. (2019). A Serendipitous Research Paper Recommender System. International Journal of Business and Management Studies, 11(1), 39-53.

24. Maluleke, W., Dlamini, S., \&Rakololo, W. M. (2019). Betrayal Of A Post-Colonial Ideal: The Effect Of Corruption On Provision Of Low-Income Houses In South Africa. International Journal of Business and Management Studies, 11(1), 139-176. 